DOI : $10.22199 / \mathrm{S} 07160917.1982 .0002 .00003$

\title{
PERFIL DEL ALUMNO DE LOS CURSOS DE MATEMATICA EN LOS PRIMEROS SMESTRES EN LA UNIVERSIDAD
}

CARACTERISTICAS GENERALES DE LA MATEMATICA.

En la introducción del libro ¿Qué es la Matemática? R. Courants. Edit. Aguilar. 1964, se lee: "La Matemática, como una expresión de la mente humana, refleja la voluntad activa, la razón contemplativa y el deseo de perfección estética. Sus elementos básicos son: lógica e intuición, análisis y construcción, generalidad y particularidad. Aunque diversas tradiciones han destacado aspectos diferentes, por su síntesis lo que constituye la vida, la utilidad y el supremo valor de la ciencia matemática".

En el capítulo La Matemática, objeto de cultura y herramienta de trabajo, "Las grandes corrientes del Pensamiento Matemático" Eudeba 1962, se comenta: "La Matemática es una disciplina del espíritu, la más rigurosa de todas. 
En ella el más o menos es inadmisible. Por lo tanto, se corre mucho menos que en otros dominios el riesgo de ilusionarse sobre las propias fuerzas. No puede haber matemáticas sin esfuerzo". "La investigación matemática, por modesto que sea su objeto, fuerza la atención hasta un punto tal que impide toda divagación perezosa del espíritu, tan común entre los adolescentes. Es tan absorbente que el problema: ien qué sueñan los jóvenes?, no se presenta para los aprendices de matemáticas" "E1 lenguaje matemático obliga a una gimnasia intelectual sumamente intensa. La Matemática desarrolla también la imaginación, pero le impide vagar más allá de los límites de una lógica rigurosa: sin intuición es imposible resolver el menor problema de geometría que no sea del tipo "demostrar que ...", con el resultado dado de antemano".

En el mismo libro mencionado en el párrafo anterior, en el capítulo La Arquitectura de las Matemáticas, se destacan las siguientes ideas: "Todos saben que el carácter externo de la Matemática es presentarse bajo el aspecto de esa "larga cadena de razones" de la que habla Descartes. Toda teoría matemática es un encadenamiento de proposiciones que se deducen una de otras conforme a las reglas de una lógica que, en lo esencial, es la codificada desde Aristóteles bajo el nombre de "lógica formal" convenientemente adaptada a los fines particulares del matemático. Pero, todo matemático sabe, además, que no se comprende verdaderamente una demostración en tanto uno se limite a verificar paso a paso la corrección de las deducciones que figuran en ella, sin tratar de captar claramente las ideas que llevaron a construir esta cadena de deducciones con preferencia a cualquier otra. E1 método axiomático halla su punto de partida en la convicción de 
que si las matemáticas no son un encadenamiento de silogísmos que se despliegen al alzar, tampoco son una colección de artificios más o menos astutos, hechos de aproximaciones fortuitas, en las que triunfa la pura habilidad técnica".

La otra característica del método axiomático es que los objetos matemáticos no tienen una significación como cosas sustanciales en sí. No es necesario entender 10 que son, sino lo que interesa es como se usan, cuáles son sus propiedades y relaciones de esos objetos. Esto es 10 que define a la Matemática como ciencia abstracta, lo que implica que todo conocimiento matemático trascienda al hombre, al espacio y al tiempo.

Una teoría matemática en el proceso de ser inventada aparece como si perteneciera a una ciencia experimental, inductiva, pero al estar completa es que aparece como sistemática deductiva, abstracta. Los matemáticos van 1ibrando una teoría matemática del método inductivo y del método experimental.

\section{COMENTARIOS.}

En los párrafos anteriores se han dado características generales de la Matemática y en todo proceso de enseñanza-aprendizaje de ella, cualquiera sea su nivel, hay que tenerlas presentas. El énfasis que se le dé, dependerá de este nivel. No se puede enseñar Matemática mediante métodos ajenos al ser de ella. A medida que pasan los años de un alumno, la enseñanza debe ir aproximándose cada vez más a la forma como se trabaja en Matemática. Este ir aprendiendo a trabajar en ella es continuo y tiende a hacerse más completo, es decir, el alumno va adquiriendo paulatina- 
mente habilidad en la operatoria, poniendo en juego las facultades inventivas, acostumbrándose a interesarse de comprender no sólo la solución de un problema sino también los motivos y el procedimiento de la solución. Esta metodología de acercarse en forma ascendente a las características de la Matemática es un proceso largo y va dependiendo de la edad mental del estudiante, pero en casa estado se puede hacer razonar al alumno y esto es lo importante, pues en el razonar se emplea la lógica. Si al alumno se le hace dedicar su tiempo sólo a ejercitar en operaciones rutinarias, no sólo no oirá aprendiendo a conocer la Matemática, sino que perderá interés por ella, y lo más triste es que pueden ser jóvenes con condiciones naturales 10 que sean afectados. E1 razonar, por 10 demás, contribuye a la disciplina intelectual.

Una cualidad que debe ir desarrollando un futuro estudiante universitario, es el de ser capáz de fijar su interés por largo tiempo, en alguna situación que requiere esfuerzo mental. Esta cualidad se cultiva, se estimula, por lo general no se dá por sí sola, ni se adquiere en poco tiempo, es de años de práctica.

E1 estudiante debe 1legar a la Universidad con un cierto entrenamiento en la capacidad de comunicar ideas científicas. Con lo que respecta a Matemática, el futuro universitario tiene que haber sido entrenado junto con operar correctamente con operaciones aritméticas y algebraícas elementales, también en leer y escribir en matemática. En esto último contribuye mucho la Geometría Plana tratada axiomáticamente y la técnica de plantear y resolver problemas.

E1 perfil del joven que ingresa al ler. año de 
carreras del área de la Ingeniería, Pedagogía en Matemática y Licenciatura en Matemática es:

- Debe ser disciplinado intelectualmente.

- Poseer conocimientos matemáticos básicos pero fundamentales.

- Tener condiciones naturales para estudiar Matemática.

Disciplina Intelectual:

- Capacidad para razonas (capacidad para expresar ideas.)

- Capacidad para fijar la atención por largo tiempo en alguna situación.

Conocimientos Básicos:

- Operar correctamente con operaciones aritméticas.

- Operar correctamente con operaciones algebraicas elementales.

- Entrenado en la técnica de plantear y resolver problemas.

- Tener un cierto entrenamiento en el método axiomático.

Respecto a las condiciones naturales éstas de nada valen sin una disciplina intelectual y sin conocimientos básicos.

En los dos primeros semestres de Universidad, es alto el número de alumnos que fracasan en sus asignaturas 
de Matemática. Entre las causas están:

- E1 no poder expresar las ideas que conducen a la solución de un ejercicio o problema: Es frecuente observar en las pruebas desorden en la redacción, se hace difícil al profesor seguir el orden en que el alumno desarrolló su solución. Es común que los alumnos no empleen las hipótesis o alguna de ella y las explicaciones que dan están fuera de toda sistematización, basadas en supuestos que no están relacionados con lo que se está resolviendo. Cuando se muestra la prueba a un alumno, éste da una larga explicación de lo que quiso hacer, pero está en su mente $y$ no en el papel. Incluso hay alumnos que teniendo las ideas en su mente no sabe como empezar a escribirlas y dejan el ejercicio respectivo en blanco, sin resolverlo. Por supuesto que otros no contestan porque simplemente no han estudiado para la prueba.

- Dificultad en la operatoria aritmética y alge braíca elemental: Todos los alumnos conocen la operatoria, pero también es frecuente encontrarse con jóvenes que tienen dificultad con ella, ya sea en la rapidez como en la comprensión de las propiedades, y sobre todo en la aplica ción de estas propiedades a ejercicios de rutina.

- Poco entrenamiento en la técnica para plantear y resolver problemas: La mayoría de los alumnos están más acostumbrados a ejercicios de rutina, que a aquellos que exigen un esfuerzo mental más intenso, es decir, que plantean una situación nueva. En las pruebas en que figuran problemas que no están en las guías de ejercicios, el fracaso es alto. Cuando aparecen en una prueba ejercicios de la guía el resultado es un poco mejor que en el caso ante- 
rior, pero no tanto. Una seria dificultad para gran númerode alumnos es no tener habilidad para ubicarse en una situación, para determinar los antecedentes de ella (datos o hipótesis), las relaciones que existen entre estos datos y determinar el procedimiento para llegar a la solución.

- La falta de disciplina intelectual: Una cualidad necesaria del estudiante universitario es tener el hábito de estudiar en forma continua y por largo tiempo. De tener la capacidad de sacrificar distracciones y días feriados. Es el requisito más necesario, pues, si están déviles otros hábitos o condiciones, con un esfuerzo sostenido en el estudio, se las puede ir adquiriendo, quizás no como debiera ser, pero lo suficiente para obtener un éxito mediano (mínimo) en el conjunto de las asignaturas. En cada asignatura universitaria se deben tratar muchos temas $y$ hay que tratarlos todos, pudiendo no verse algunos detalles, pero sí lo esencial de cada unidad. Luego no hay mucho tiempo para ponerse a esperar a que todos los alumnos aprendan. No hay plan de estudios, programas o metodologías que valgan, sin el esfuerzo intelectual intenso del alumno.

- La perenne falta de material bibliográfico: La cantidad de material bibliográfico existente no alcanza a cubrir la necesidad de él, por parte de los alumnos. Pero quizás éste sea el mal menor, comparado con las causas anteriores.

CONCLUSION.

A los primeros cursos de Matemática de la Uni- 
versidad, el alumno debe ingresar con las habilidades y hábitos que se han descrito. No se puede pretender recién a comenzar a formarlos en esta primera etapa. En realidad a partir de ella, se les pone en uso con mayor intensidad. En palabras del Dr. Roberto Barahona Silva, uno de los fundadores (organizadores) de CONICYT, se escucha: "Los estudiantes deben egresar de Enseñanza Media con el afán de saber, con la tendencia a entender por propia iniciativa los problemas que se les presentan, con el deseo de leer y conocer las opiniones ilustradas de las generaciones precedentes". (Diario La Tercera, 15 de Agosto -1982. Segundo Cuerpo).

E1 paso de la Enseñanza Media a la Universitaria debe ser continuo, y no un salto discontinuo. Mientras se mantenga esta discontinuidad, el porcentaje de fracaso se mantendrá en los dos primeros semestres de Universidad. Una manera de elevar el rendimiento es mediante la recuperación de una parte de los alumnos que fracasen en el ler. Semestre o en el ler. año. El rendimiento no se elevará sólo mediante reglamentos severos, pues un reglamento no forma hábitos. Ellos pueden adquirirse ejercitándolos y para esto se requiere tiempo.

Finalmente, sería de gran interés que se hiciera un estudio sobre el rendimiento en Matemática en los dos primeros semestres en cada carrera de cada Universidad del país en los últimos cinco años (u otro período), incluyéndose el rendimiento de algunas carreras de ciertas Universidades de algunos países Latinoamericanos, de Estados Unidos y de algunos países europeos. Con este antecedente debería efectuarse un Congreso so- 
bre el proceso enseñanza aprendizaje de la Matemática en el ler. año universitario. Esta labor podría ser efectuada por algún organismo tal como Consejo de Rectores, CONICYT, Ministerio de Educación o Sociedad de Matemática de Chile. Entre las preguntas a responder están : ¿Hasta qué punto interesa el porcentaje de rendimiento? ¿Interesa la calidad y/o cantidad?. Si interesa la cantidad ¿Cuál es su monto? ¿Cuál es la metodología para 1legar a un porcentaje de rendimiento?, etc. 